\section{Aedes Mosquito Larva in the Hospital: A Note From Thailand}

To the Editor-Mosquito-borne infections are increasingly important. To control mosquito-borne infections, mosquito control is needed. The Aedes mosquito is an important vector for many diseases including the Zika virus and dengue fever. The survey of Aedes mosquito larva is a routine public health practice in tropical countries. ${ }^{1}$ Mosquito larvae can be found in many urban buildings. Hospitals can also be the setting of habitat for the Aedes mosquito, but this fact is mentioned infrequently. Here, I report and discuss the data from an Aedes mosquito larva survey in hospitals in an endemic area of Thailand (western region, 7 provinces) during the rainy season, May-June 2015. Overall, 30 hospitals were surveyed, and Aedes mosquito vector larva were detected in 16 hospitals (53.3\%). The percentage of water-holding containers infested with larvae ranged from 0 to 30 . The number of larvae-positive containers per 100 containers inspected at hospitals, the Breteaux index, ranged from 0 to 20.59. Based on these data, many hospitals can be considered the source for Aedes-borne infectious disease. Indeed, an important role of the medical center is to provide health care to and promote health within the community. Mosquito control in the hospital is usually a forgotten issue, but it can pose a significant problem if there is a healthcare-associated outbreak of mosquito-borne infection in the region. In the era of emerging and remerging mosquito-borne infections (eg, Zika virus infection, dengue and others), mosquito control in the hospital is necessary ${ }^{2}$ and represents an emerging and important issue in the field of infection control and hospital epidemiology.

\section{Viroj Wiwanitkit, MD}

Affiliation: Chulalongkorn, University Bangkok, Bankok, Thailand.

Address correspondence to Professor Viroj Wiwanitkit, MD, Department of Laboratory Medicine, Faculty of Medicine, Chulalongkorn, University Bangkok, Bankok, Thailand 10330 (wviroj@yahoo.com).

Infect Control Hosp Epidemiol 2016;37:1519

(c) 2016 by The Society for Healthcare Epidemiology of America. All rights reserved. 0899-823X/2016/3712-0024. DOI: 10.1017/ice.2016.212

\section{REFERENCES}

1. Benelli G, Mehlhorn H. Declining malaria, rising of dengue and Zika virus: insights for mosquito vector control. Parasitol Res 2016;115:1747-1754.

2. Wiwanitkit V. Zika virus infection: control and prevention. J Chin Med Assoc 2016;79:409.

\section{Can Fecal Microbiota Transplantation (FMT) Eradicate Fecal Colonization With Vancomycin-Resistant Enterococci (VRE)?}

To the Editor-Recently, fecal microbiota transplantation (FMT) has been attempted to eliminate colonization with multidrug-resistant organisms (MDROs), and case reports have shown considerable success. ${ }^{1}$ In addition, FMT was effective for reducing antibiotic resistant genes in patients with recurrent Clostridium difficile infection (CDI), ${ }^{2}$ which may have a significant role in MDRO decolonization. However, publication bias could exist against studies with negative findings for MDRO decolonization, and clinical evidence for extending the application of FMT remains sparse. We performed FMT to eradicate long-term vancomycin-resistant enterococci (VRE) colonization in 3 patients. Prolonged VRE colonization was documented by repeated rectal swab cultures (positive VRE on at least 4 consecutive swabs taken 1 week apart). Of these 3 patients, 2 had recurrent CDI and were treated with oral metronidazole and vancomycin (Table 1 , cases 1 and 2). Another patient (case 3) remained in the hospital for isolation purposes due to VRE carriage after completion of treatment. Vancomycin resistance was confirmed using chromogenic agar and polymerase chain reaction. All cases were Enterococcus faecium with the vanA gene. Voluntary informed consent was obtained for FMT. Stools were donated by the patient's granddaughter (case 1, 18 years old), daughter (case 2, 45 years old), and son (case 3, 50 years old), respectively. The donors were healthy and no problems were identified on pre-donation screening tests; they were all negative for stool VRE. Oral vancomycin treatment was discontinued on the day before FMT (case 1 and 2), and the donor stool $(100 \mathrm{~g})$ in normal saline $(200 \mathrm{~mL})$ was transplanted to the patient via retention enema after environmental disinfection. All patients were able to retain the infusate for at least 1 hour, and there were no adverse events. In case 3, a second FMT was performed 1 day after the first FMT. All antibiotics were stopped after the FMT was conducted except in case 2, in which the patient developed pneumonia 15 days after FMT and piperacillin-tazobactam was given for 2 weeks. Patient characteristics and outcomes are summarized in Table 1. Patients 1 and 2 experienced resolution of CDI symptoms without recurrence during admission. After transferring to other facilities, 2 patients were lost to follow-up. We did not recruit additional patients because FMT did not shorten the duration of VRE carriage in these 3 patients.

Our data are supported by the Jang et $\mathrm{al}^{3}$ case report in which FMT was performed twice to control refractory CDI. Their patient was also colonized with VRE and, despite 